\title{
ON THE ORIGIN OF CENTRAL PEAKS IN THE CRATER FORMATIONS FILLED WITH MELT AFTER IMPACTS
}

\author{
L. Ǩ̌ IVSKÝ \\ Astronomical Institute, Ondřejov, Czechoslovakia
}

\begin{abstract}
An attempt is made at explaining the successive origin of the central peaks and terrace ringwall in the crater formations filled with melt after impacts.
\end{abstract}

In the course of experiments with freezing water containing an organic fine and coarse substance in a vessel at temperatures of -10 to $-15^{\circ} \mathrm{C}$ and freezing distilled and undistilled water in a vessel at temperatures of -3 to $-10^{\circ} \mathrm{C}$ the forming of the shape of the surface and the structure of the internal content were investigated. Description of one of the experiments: Freezing of undistilled water in an open glass vessel (height $12 \mathrm{~cm}$, width $7 \mathrm{~cm}$, water level at a height of $9 \mathrm{~cm}$ ). The initial temperature of the water was $\sim 30^{\circ} \mathrm{C}$, the ambient temperature -5 to $-10^{\circ} \mathrm{C}$, and the temperature of the base on which the vessel was standing was $-5^{\circ} \mathrm{C}$. Conditions after about $10 \mathrm{hr}$ of exposure: the water froze in a thicker layer at the bottom of the vessel, the walls but less at the surface and remained clear; in the central part of the water the space with the unfrozen water had the shape of a smaller egg filled in its upper part with an air bubble. Surface profile: central peak of a height of about $0.5 \mathrm{~cm}$ with a broader base. Conditions after about $20 \mathrm{hr}$ of exposure: the water in the vessel froze completely; around the vertical axis of the vessel inside the ice there are regions of fine frozen-in bubbles pointing towards the axis and slightly upwards, and they are also ordered along the upper part of the axis under the surface where they point towards the central surface peak; the surface kept its previous shape.

The central peak is clearly due to the initial freezing of the boundary regions of the water which has the shape of a cylinder, and at his time gaseous components are released and pressed out into the central liquid regions. The latter freeze a little later. The volume pressure from the sides causes the unfrozen water in the region of the vessel axis to be compressed vertically, and the liquid with gaseous cavities is pushed up through the narrow central region to the weakly frozen surface were it also freezes. The central peak is formed by liquid eruptions of longer duration within a narrow space in the centre of the freezing surface, where the gaseous components are pressed out and released. These are in fact trivial processes occurring during the gradual nonuniform freezing of water in vessels of cylindrical or bowl-like shapes, when volume and structural differentiation occurs. These processes can be compared with the processes of solidification of melted rocks in craters, when the melted rocks after the impact and explosion of large meteors, or after an effusion of subsurface origin as a result of perturbation by impact. The mechanism described, due to non-uniform 
solidification and volume differentiation with the forming of the central peak would of course be applicable if water were present on the Moon in larger volumes in the solidifying and crystallizing melt and provided the melt would go down to a sufficient depth. The water could even be at the lower horizons, at the bottom of craters. This possibility has not been excluded yet. Analogous formations based on this "water mechanism" are also known from the Earth's surface. The cooling and solidifying connected with crystallization progressing from the edges to the centre of lunar craters may form an analogous mechanism of pressing out escaping gaseous and liquid constituents into the central regions, where extrutions would occur in the centre of the surface above the slowly solidifying surrounding surface; a cone with a 'funnel' would be formed through which the gases from the lower levels would escape at irregular intervals.

The size of the conical peak above the surface would depend on a number of parameters, e.g., on the depth of the region of the molten rocks, on the ratio of the diameter of the melt pool to its depth, on the physical and chemical properties of the melt (on the structural differentiation during the process of solidification), on the ambient temperature of the rocks around the crater, and on the temperature gradient along the radius.

It is also possible that the volume of the solidified melt itself would not have to be larger in comparison to the volume of the original liquid melt in order to form structural differentiation leading upto the formation of the central regions with escaping gases as is the case in the water experiments.

\section{2}

Should the melt be nearly waterless (and should there be no water even in the subsurface horizons), the process of structural differentiation will be applicable together with the dynamics within the spatially non-uniformly solidifying melt as a result of gravitational differentiation, which is well known, e.g., from the solidifying of molten basalts under laboratory conditions. The volume of a solid crystallized basalt is $3-10 \%$ smaller than the volume of the original melt. In the solidifying volumes of spherical shapes large cavities are formed in the centre (Kopecký, 1971).

Processes would occur on the Moon which are illustrated in sequence on the vertical section of the crater in the diagrams attached.

Figure 1a: (a) - regions of initial solidification of a basalt melt which was created by heating on impact or by partial effusion of the still liquid materials from below; heat losses by radiation and transfer after impact are the largest in these regions.

Figure $1 \mathrm{~b}:\left(\mathrm{a}_{1}\right)$ - the heavier sinking blocks of solidified and half-solid basalt melt; (b) - the hottest central regions where the gaseous constituents are concentrated. This lighter region has the tendency to ascend. The gaseous bubbles join up and larger and larger regions filled with gas are formed with a tendency of a more rapid ascension towards the surface. The gases escape from the bubbles mostly in the central regions on the surface (c). The gaseous bubbles captured temporarily or permanently by the 
top layer may form domes (d). Part of ascending melt flows out to the sides where it partly substitutes the sinking solid blocks. The sinking of the blocks $\left(a_{1}\right)$ is the largest on the inside of the crater, and faults occur on the outer sides of the blocks. The outer edges of these slanting blocks form new lower inner edges of boundary ringwalls (e). This effect may be repeated several times as a result of the irregular decreases and turning of the solid boundary blocks around horizontal axes. The overall height of the surface gradually decreases (as a result of the total diminution of the volume of the solidifying melt). Cracks appear on the surface crust (f) and this is where the melt extrudes and the gas bubbles escape from the lower regions (b) above the sinking parts of the solid blocks.
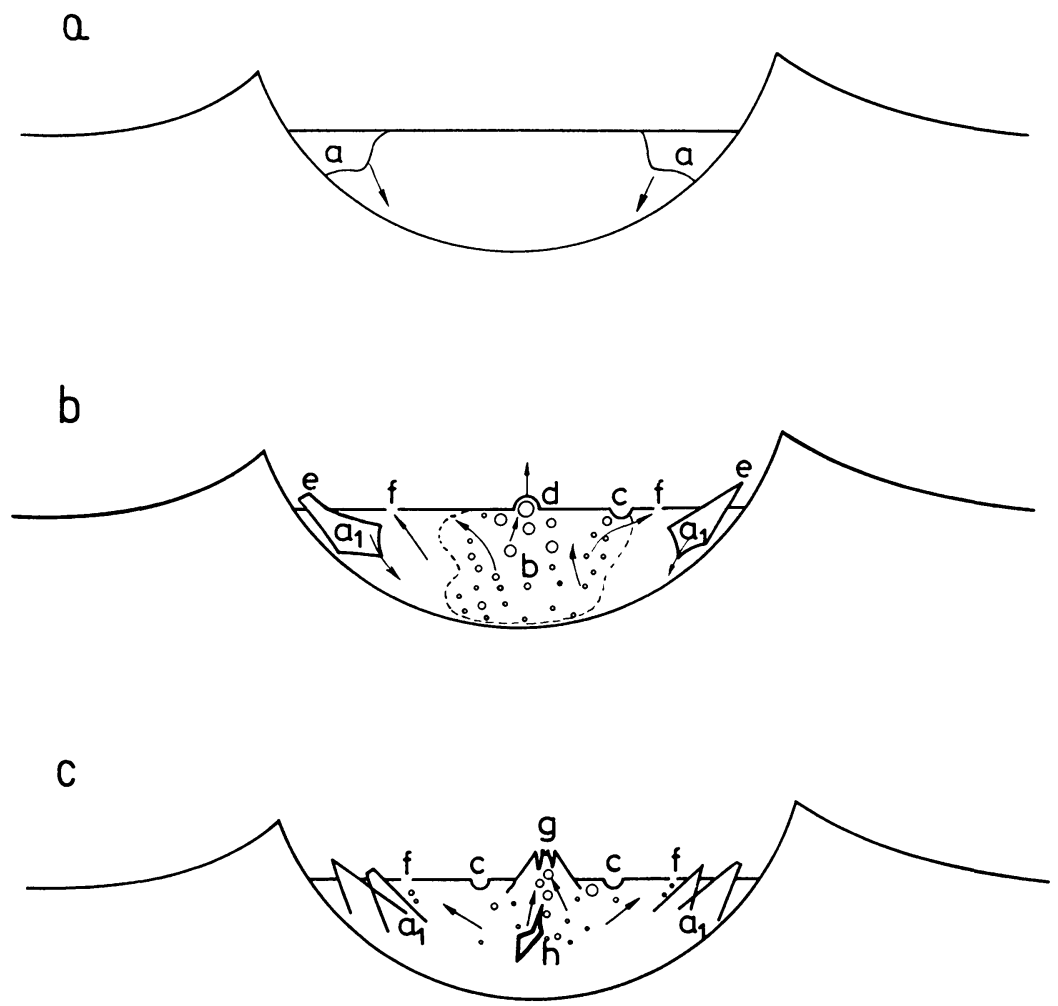

Fig. 1.

Figure 1c: If comparatively large bubbles escape from the central part of the melt towards the surface the surface crust are sometimes broken up and lifted (g). The central regions of this formation can then be filled by the melt coming from below. This process may be repeated several times, if several hot gaseous regions were formed and if the depth of the bowl-shaped melt region was sufficiently deep in comparison to the diameter of the crater. Impulses from neighbouring impacts causing moon- 
quakes could be responsible for the formation of the central peaks (by rupturing the surface crust and causing the melt to flow out and gases to escape).

In the last phase also the central part solidifies. At this time cracks and cavities with little gas filling are formed in the internal parts $(\mathrm{h})$, the cracks may interconnect bubbles filled with gases earlier captured by the semi-liquid melt. These processes may take thousands and tens of thousands of years, and sporadic extrusions and escapes of gases through the cracks from the cavities may go on for hundreds of thousands and millions of years. This need not be volcanism proper, but the processes could be called 'quasivolcanic', created in a melt within the region of crater after impact.

These process of differentiation and dynamics of a solidifying melt after large impacts may be used to explain the origin of the terrace-like inner boundaries of the crater ringwalls of some craters, the central regions with the domes or smaller craters (after gas escape), or of the central peaks (including the peak crater).

Should this analogy prove to be correct the central peaks in the crater formations would be due to the process of successive solidification of melts of limited volumes in the surrounding environment with temperature well below the point of solidification, and they would not have to represent an argument for the volcanic theory of the origin of some of the craters; some 'quasivolcanic' processes occurring later would thus evolve in the regions of the melt of bowl-like shape after impact.

These resultant effects are nearly identical with the effects which are caused by the solidification of melts which contain a high percentage of water, when the solidified parts could have a larger volume than the original melt. The physical dynamic process is however different in both cases considered; the measure of interconnection of both processes ( 1 and 2 ) may differ under lunar conditions.

Possible relations between meteorite impact and igneous petrogenesis were discussed also by French (1970).

If the volume of the solidified melt is neither larger nor smaller than the volume of the original melt, larger central peaks nor multiple terrace-like edges on the inner sides of the ringwalls would not be created with the exception of structural differentiation. Neither could gravitational differentiation and dynamics evolve properly in these cases when the crater deep is shallow (as compared to the crater diameter at melt surface level). The differentiation will also be conditioned by a certain sufficient temperature difference between the initial temperature of the melt lake and the ambient lower temperature of the surface rocks in the vicinity of the crater.

\section{Acknowledgements}

The author is indebted to Mrs B. Křivská (Ondřejov) for pointing out the phenomena connected with the solidification of liquids in limited volumes, to Prof. V. Guth 
(Astronomical Institute Ondřejov) for his great interest and help and Ing. J. Valenta (Basalt Melting Works Stará Voda) for consultations and some of the experiments.

\section{References}

French, B. M.: 1970, Bull. Vulcanologique 34, 466.

Kopecký, L.: 1971, private communication. 